\title{
Progesterone Induces Scolex Evagination of the Human Parasite Taenia solium: Evolutionary Implications to the Host-Parasite Relationship
}

\author{
Galileo Escobedo, ${ }^{1}$ Ignacio Camacho-Arroyo, ${ }^{1}$ Olivia Tania Hernández-Hernández, ${ }^{1}$ \\ Pedro Ostoa-Saloma, ${ }^{2}$ Martín García-Varela, ${ }^{3}$ and Jorge Morales-Montor ${ }^{2}$ \\ ${ }^{1}$ Departamento de Biología, Facultad de Química, Universidad Nacional Autónoma de México, AP 70228, 04510 México, DF, Mexico \\ ${ }^{2}$ Departamento de Inmunología, Instituto de Investigaciones Biomédicas, Universidad Nacional Autónoma de México, \\ AP 70228, 04510 México, DF, Mexico \\ ${ }^{3}$ Instituto de Biología, Universidad Nacional Autónoma de México, 04510 México, DF, Mexico
}

Correspondence should be addressed to Jorge Morales-Montor, jmontor66@biomedicas.unam.mx

Received 30 July 2009; Accepted 14 September 2009

Academic Editor: Luis I. Terrazas

Copyright (C) 2010 Galileo Escobedo et al. This is an open access article distributed under the Creative Commons Attribution License, which permits unrestricted use, distribution, and reproduction in any medium, provided the original work is properly cited.

Taenia solium cysticercosis is a health problem in underdeveloped and developed countries. Sex hormones are involved in cysticercosis prevalence in female and male pigs. Here, we evaluated the effects of progesterone and its antagonist RU486 on scolex evagination, which is the initial step in the development of the adult worm. Interestingly, progesterone increased T. solium scolex evagination and worm growth, in a concentration-independent pattern. Progesterone effects could be mediated by a novel T. solium progesterone receptor (TsPR), since RU486 inhibits both scolex evagination and worm development induced by progesterone. Using RT-PCR and western blot, sequences related to progesterone receptor were detected in the parasite. A phylogenetic analysis reveals that TsPR is highly related to fish and amphibian progesterone receptors, whereas it has a distant relation with birds and mammals. Conclusively, progesterone directly acts upon T. solium cysticerci, possibly through its binding to a progesterone receptor synthesized by the parasite.

\section{Introduction}

Cysticercosis, caused by the metacestode stage of Taenia solium, is a serious health and veterinary problem in many developing countries [1-3]. In humans, T. solium cysticerci cause neurocysticercosis, which affects $\sim 50$ million people worldwide, and it has been recently considered as an emergent disease in the USA [4]. T. solium also infects pigs, its intermediate host, leading to major economic losses $[5,6]$.

When humans ingest undercooked contaminated pork meat, the adult worm develops in the small intestine. After two months of asymptomatic infection, this tapeworm starts producing thousands of eggs that, once released with the stools, can contaminate the environment, infecting pigs (rapidly differentiating into cysticerci mainly in the muscle) and humans (where most severe symptoms are observed due to the presence of cysticerci in the brain) $[1,7]$.

Thus, maintenance of the parasite's life cycle depends on the adult tapeworm development [8]. In fact, even in communities which do not rear or consume pigs, human neurocysticercosis can be found, because of the presence of a tapeworm carrier $[9,10]$. Furthermore, tapeworm development in turn depends on scolex evagination, the initial step through which a single cysticercus becomes an adult parasite with capability of producing infective eggs [11].

Recent information reveals that sex hormones can affect the course of a parasite infection [12-16], as in the case of taeniasis/cysticercosis [17-19]. Moreover, frequency of $T$. solium pig cysticercosis is increased during pregnancy, when there is a significant increase in progesterone levels $[19,20]$. 
It has also been demonstrated that castration in naturally infected male boars, induces an increase in the prevalence of cysticercosis, which highlights the possible role of host androgens to restrict parasite establishment and estrogens to facilitate it [19].

Furthermore, Taenia crassiceps (a close relative of $T$. solium) has shown to be affected by in vivo and in vitro sex steroid treatment. Specifically, $17 \beta$-estradiol increases the reproduction of $T$. crassiceps cysticerci in vitro, while testosterone or dihydrotestosterone decreases it [21]. When castrated mice are treated with $17 \beta$-estradiol, the number of parasites as well as their infective capacity increases up to $200 \%$ [22, 23] meanwhile progesterone has the opposite effect in castrated mice of both sexes: a decrease in the parasite loads of almost 100\% [24].

Since an mRNA sequence similar to that of estrogen receptor has been found in T. crassiceps [21], it is possible that the direct effect of estradiol on T. crassiceps reproduction could be due to its binding to this receptor [21]. In fact, numerous sex steroid actions in vertebrates are mediated by the binding to their nuclear receptors, which in this form regulate gene expression, as in the case of estrogens [25], androgens [26], and progesterone [27]. The latter interacts with two main progesterone receptor (PR) isoforms [28], whereas other hormones such as androgens only have one specific receptor [26]. Interestingly, a similar mechanism could occur also in parasites $[21,29,30]$.

Steroid hormone effects are not restricted only to cestode parasites but also to nematodes such as Ancyclostoma dudodenale, whose number of larval and adult stages is increased by sex steroid hormones in several organs of mice [31]. Moerover, adult and muscle larvae of Trichinella spiralis are increased in ovarectomized female rats [14], suggesting that estrogens are restrictive factors for parasite establishment, while androgens should play a permissive role to the infection. Concomitantly, trematode parasites are also affected by sex steroids. For instance, in vitro, testosterone has an antifecundity effect upon male and female Schistosoma mansoni adult worms [29], as well as dehydroepiandrosterone, which in vitro reduces the viability and oviposition of Schistosoma mansoni [32].

As it can be seen, direct effects of sex steroids upon helminth parasites (cestodes, nematodes, and trematodes) are not unusual. In fact, previous results suggest that these pathogens are not only directly affected by hormones, but they have also developed several strategies to exploit the host's endocrine microenviroment [33,34], which include degradation of host proteins as an alternative source of aminoacids [35], development of parasitic-sex steroid receptors $[29,30]$, and cross-activation of signal transduction pathways [36, 37].

Taking into consideration this information, the aim of the present study was to explore the role of progesterone on T. solium cysticerci development, evaluating its in vitro effects on scolex evagination and adult worm growth, key processes in the maintenance of the infectious cycle in pigs and humans. The in vitro effect of progesterone on T. solium was studied through pharmacological (the use of RU486, a progesterone antagonist which binds to intracellular PR) and molecular (RT-PCR, western blot, phylogenetic analyses) approaches, in order to figure out the mechanism of progesterone actions in the parasite.

\section{Materials and Methods}

2.1. Obtention of Parasites. T. solium cysticerci were dissected from the muscle of infected pigs, which were euthanized at the Veterinary School of the Universidad Nacional Autónoma de México. The method was previously evaluated by the University Animal Care and Use Committee to ensure compliance with international regulations and guidelines. The fibrous capsule that surrounds each parasite was carefully separated with the use of a dissection microscope. Once dissected, cysticerci were placed in tubes containing sterile PBS (1X) supplemented with $100 \mathrm{U} / \mathrm{mL}$ of antibiotics-fungizone (Gibco,Grand Island, NY, USA) [38]. Samples were centrifuged for 10 minutes, at $800 \mathrm{~g}$ at $4^{\circ} \mathrm{C}$ and the supernatant was discarded. Pellets containing cysticerci were incubated in Dulbecco's Modified Medium (DMEM) without fetal serum supplementation (Gibco, BRL, Rockville, Md, USA). They were then washed by centrifugation 3 times for 10 minutes at $800 \mathrm{~g}$ with DMEM. After the final wash, viable parasites (complete and translucent cystic structures) were counted using a binocular microscope.

\subsection{Progesterone and RU486 Dose-Response-Time Curves.} Culture grade progesterone and RU486 were obtained from Sigma (Sigma-Aldrich, USA). For in vitro tests, progesterone-water soluble (powder cell culture tested, Sigma-Aldrich, USA) was dissolved in DMEM free-serum culture medium, while RU486 was dissolved in pure ethanol (Sigma) to the desired stock concentration, and sterilized by passage through a $0.2 \mathrm{~mm}$ millipore filter. For concentrationresponse curves, the experimental design was as follows, using four wells per treatment: (a) progesterone vehicle (only DMEM); (b) RU486 vehicle (ethanol at the final concentration of $0.06 \%$ per well); (c) progesterone at $0.06,0.25,2.5$, 3.175 , and $63.5 \mu \mathrm{M}$; (d) RU486 (at the same concentrations of progesterone); and (e) a combination of progesterone and RU486 in all concentrations described before. For timeresponse curves, cysticerci from all treatments were cultured during 20 days, with daily inspections of scolex evagination and worm length. Culture wells contained $5 \mathrm{~mL}$ of DMEMmedium and were incubated at $37^{\circ} \mathrm{C}$ and $5 \% \mathrm{CO}_{2}$. Progesterone and RU486 were prepared in a final volume of $100 \mu \mathrm{L}$ and added to $5 \mathrm{~mL}$ of medium in each well. From concentration-response curves of each steroid, we selected an optimal concentration for progesterone $(0.25 \mu \mathrm{M})$ and RU486 $(2.5 \mu \mathrm{M})$, to be used in the time-response curves. Culture media, as well as hormone treatments, were completely replaced every 24 hours during 20 days of culture. Scolex evagination and worm length were daily determined in all cultured cysticerci using an inverted microscope (Olympus, MO21, Tokyo) at 10X and 20X magnification. Worm length was considered as the millimetric addition of scolex, neck, and strobila. 
2.3. RNA Extraction of Cultured Cysticerci in Presence of Progesterone and RU486. Total RNA was isolated from T. solium cysticerci of each in vitro treatment as well as from uterus of mouse (positive expression control) using Trizol reagent (Invitrogen, Carlsbad, Calif, USA). In brief, cysticerci were disrupted in Trizol reagent $(1 \mathrm{~mL} / 0.1 \mathrm{~g}$ tissue) and $0.2 \mathrm{~mL}$ of chloroform was added per $\mathrm{mL}$ of Trizol. The aqueous phase was recovered after 10 minute of centrifugation at $14000 \mathrm{~g}$. RNA was precipitated with isopropyl alcohol, washed with $75 \%$ ethanol, and redissolved in RNAse-free water. RNA concentration was determined by absorbance at $260 \mathrm{~nm}$ and its purity was verified after electrophoresis on $1.0 \%$ denaturing agarose gel in presence of $2.2 \mathrm{M}$ formaldehyde.

\subsection{T. solium Progesterone Receptor (TsPR) Gene Amplifi-} cation. Total RNA from all treated cysticerci was reversetranscribed followed by specific PCR amplification of the putative TsPR by using one specific pair of primers designed to amplify corresponding fragments of the DNA-binding domain (one of the most conserved regions of all PR sequenced genes reported in the NIH Gene Data Bank). $\beta$ actin was used as a control gene of constitutive expression, as we have previously described [21,39]. Primer sequence of PR was: sense $5^{\prime}$-GGAGGCAGAAATTCCAGACC and antisense $5^{\prime}$-GACAACAACCCTTTGGTAGC; for $\beta$-actin, primer sequence was sense $5^{\prime}$-GGGTCAGAAGGATTCCTATG and antisense $5^{\prime}$-GGTCTCAAACATGATCTGGG. PCR products were visualized in $2 \%$ agarose gel stained with ethidium bromide. In all cases, a single-band corresponding to the expected base pair size of the amplified gene fragment was detected. Uterus from intact mouse was used as positive expression control for PR expression. TsPR expression is presented as the ratio of the optical density (OD) of the studied gene relative to the expression in the same preparation of the $\beta$-actin gene.

2.5. PCR Product Sequencing. TsPR was directly purified from the gel using a commercial kit (DNeasy Tissue Kit, QIAGEN) and sequenced. DNA sequences were determined by using a Thermo Sequenase cycle sequencing kit (Biorad) and an automatic sequencer (Model LIC-4200, Aloka Co.). Sequence data were analyzed by using DNASIS Software (Hitachi Software Engineering, Tokyo, Japan). In addition, nucleotide sequences were translated to their corresponding protein sequences by means of ExPASy Molecular Server.

2.6. TsPR Detection by Western Blot. Protein was obtained from cultured $T$. solium cysticerci. Untreated cysticerci and those treated with progesterone and/or RU486 were disrupted in Tris- $\mathrm{HCl}(1 \mathrm{~mL} / 0.1 \mathrm{~g}$ tissue), proteinase $\mathrm{K}$ (100 units $/ \mathrm{mL}$ ), and proteases inhibitor cocktail (Calbiochem). The supernatant was recovered after 15 minutes of centrifugation at $8000 \mathrm{~g}$. Protein concentration was obtained by absorbance at $320 \mathrm{~nm}$ using the Bradford-Lowry method. Total protein of T. solium ( $50 \mu \mathrm{g}$ per well) was boiled in reducing LaemmLi sample buffer, separated by SDS-PAGE (10\% acrylamide) and electro-blotted onto nitrocellulose membranes.
Membranes were blocked for $2 \mathrm{~h}$ with PBS 1X buffer $\left(137 \mathrm{mM} \mathrm{NaCl}, 2.7 \mathrm{mM} \mathrm{KCl}, 4.3 \mathrm{mM} \mathrm{Na} \mathrm{Na}_{2} \mathrm{HPO}_{4}\right.$, $1.47 \mathrm{mM} \mathrm{KH}_{2} \mathrm{PO}_{4}$ ) containing $0.25 \%$ of BSA. For protein immunodetection, membranes were subjected overnight to immunoblotting with $1 \mu \mathrm{g} / \mathrm{mL}$ of anti-PR polyclonal antibody (C-20, Santa Cruz Biotech.), diluted 1: 1500, followed by HRP-conjugated anti-rat IgG (Santa Cruz Biothecnology; diluted 1 : 5000) for 1 hour, at room temperature. Next, membranes were washed five times in 1X PBS and bands were visualized using the Enhanced ChemoLuminicensce system, according to manufacturer's instructions (Super Signal ECL, Pierce). Chemiluminiscent signals were captured on Kodak Bio-Max film, and bands were quantitatively analyzed from digitized images captured from the films with the Gel-Doc system (BioRad, Richmond, Calif, USA), using the Bio-Rad Quantity One software. Rat uterus was used as positive expression control for this experiment. The content of the protein band corresponding to the TsPR is presented as the ratio of the optical density of the studied protein relative to the content of $\alpha$-tubulin in the same preparation, used as a constitutively loading control protein.

2.7. Phylogenetic Analysis of the TsPR. The TsPR protein sequence was aligned to the $\mathrm{PR}$ protein sequences of other species (including mammals, birds, fish, one reptilian, and one amphibian) obtained from protein data sets in GenBank. Sequence alignment was done using Clustal W software [40]. Alignment of TsPR contained 88 amino acids from 12 different taxa. Phylogenetic relationships were inferred using the Neighbor joining (NJ) method. Robustness of the NJ tree was evaluated using bootstrap of 10000 replicates. The tree was drawn using RETREE and DRAWGRAM from PHYLIP [41]. The genetic differentiation between taxa was estimated using the mean character difference with the help of PAUP* $4.0 \mathrm{~b} 10$ software [42]. It is important to point out that the number of species used for the analysis was selected based on the PR sequence found in the gene data bank (for some species there is only one sequence).

2.8. Experimental Design and Statistical Analysis. Hormone dose-response-time curves were estimated in 4 independent experiments. The response variable used in statistical analyses was the total number of evaginated scolices that showed worm growth and motility in all wells of each hormone concentration and time of exposure, for every experiment. Hormones, their concentrations and times of exposure, were the independent variables. The data for the 4 replicates of each treatment were expressed as an average. Data were analyzed using one way variance analysis (ANOVA). If ANOVA showed significant differences among treatments, a Tukey Test was applied for test significance. Differences were considered significant when $P<.05$.

\section{Results}

When T. solium cysticerci were in vitro exposed to progesterone, an increase in the scolex evagination was observed in all treated parasites compared to control groups, where 


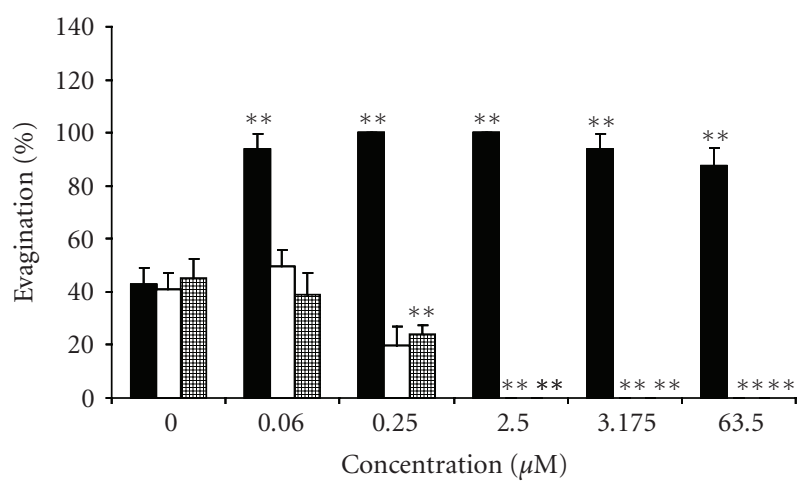

- Progesterone $\square$ RU486 + progesterone 田 RU486

(a)

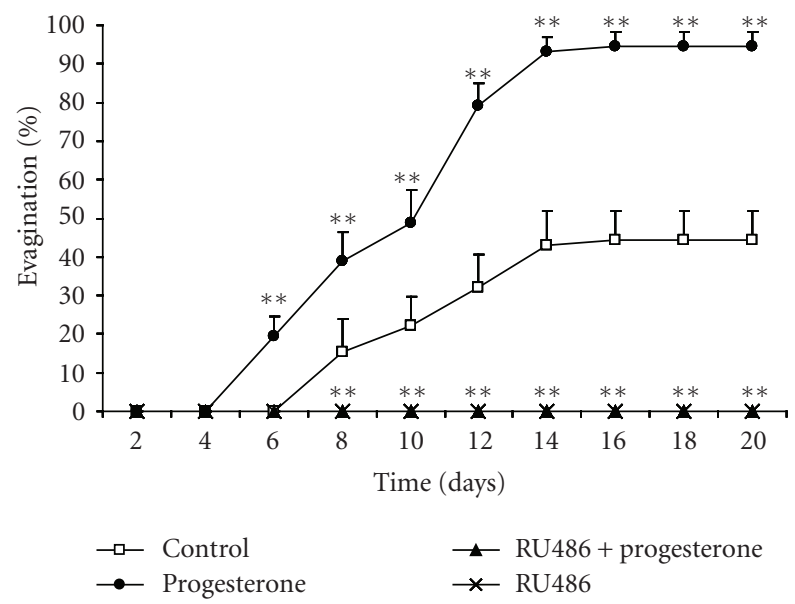

(b)

FIGURE 1: Progesterone induced scolex evagination of Taenia solium in a concentration-independent pattern (a) maintained along the time (b). On the contrary, RU486 exerted a potent concentrationdependent evagination-inhibitory effect (a), even in presence of progesterone and during the total 20 days of in vitro culture (b). In concentration-response curves (panel a), cysticerci treated with vehicle are referred as concentration zero. Data are represented as mean $+/-\mathrm{SD} ;{ }^{* *} P<.05$.

only $40 \%$ spontaneously evaginated (Figure 1). However, this evagination-promoting effect mediated by progesterone was independent of the tested concentrations (Figure 1(a)). RU486 showed a strong anti-parasite effect, since progressively inhibited scolex evagination, reaching its maximum effect at $2.5 \mu \mathrm{M}$, even in the presence of progesterone (Figure 1). Interestingly, in the case of RU486, a concentration-dependent pattern was evident, and no significant differences were observed between RU486 plus progesterone and RU486 alone-treated groups (Figure 1(a)).

Concomitantly, the evagination-promoting effect of progesterone $(0.25 \mu \mathrm{M})$ was maintained through all 20 days of in vitro culture, reaching its highest response on day 14 in culture, in relation to untreated parasites (Figure 1(b)). Consistently, when cysticerci were exposed to $2.5 \mu \mathrm{M}$ of RU486, scolex evagination was observed neither during

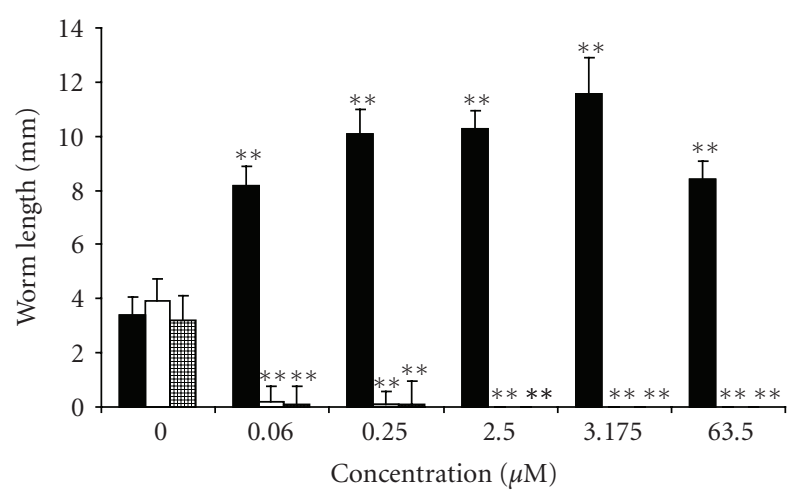

- Progesterone $\square$ RU486 + progesterone 田 RU486

(a)

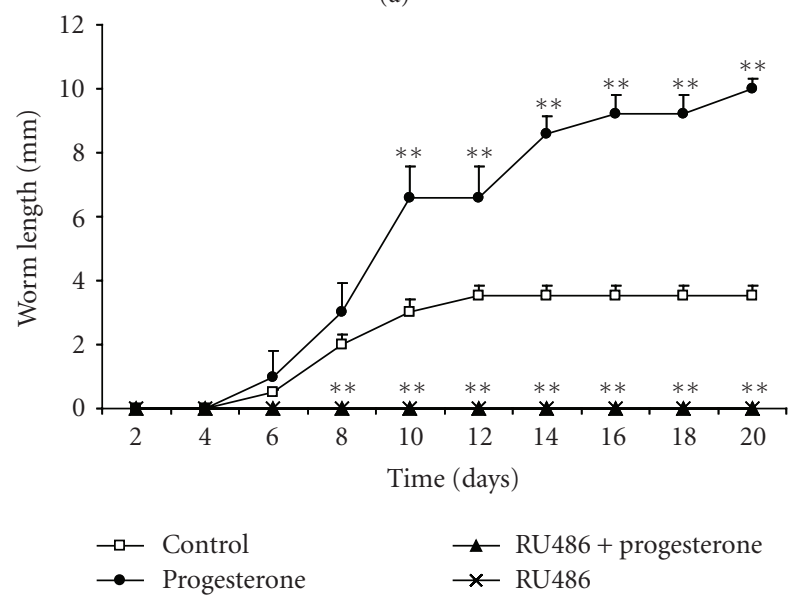

(b)

FIGURE 2: Progesterone stimulates worm growth in a concentrationindependent pattern (a), reaching its maximum effect at 20 day of in vitro culture (b). In contrast, RU486 inhibits worm development with the lowest concentration $(0.06 \mu \mathrm{M})$ (a) and during the whole culture time (b). Progesterone-treated parasites were motile and undamaged on the culture plate, typically distinct to those treated with RU486. Worm length was considered as the addition $(\mathrm{mm})$ of scolex, neck, and strobila. In the concentration-response curves (panel a), cysticerci treated with vehicle are referred as concentration zero. Data are represented as mean $+/-\mathrm{SD} ;{ }^{* *} P<$ .05 .

the first days of culture nor at the end of the process (Figure 1(b)).

It is important to mention that viability of evaginated cysticerci was verified daily by means of worm motility in the culture plate, which was constant through all days of in vitro culture. Injured parasites were recognized by a progressive internal disorganization: development of opaque areas in the tegument and loss of translucence of the vesicule (data not shown).

Progesterone also affected in vitro worm growth. From the lowest concentration $(0.06 \mu \mathrm{M})$ progesterone duplicated worm length on day 10th (measured as the addition of scolex, neck, and strobila of the developing parasite) with respect to the control group, and reached a plateau (Figure 2(a)). 


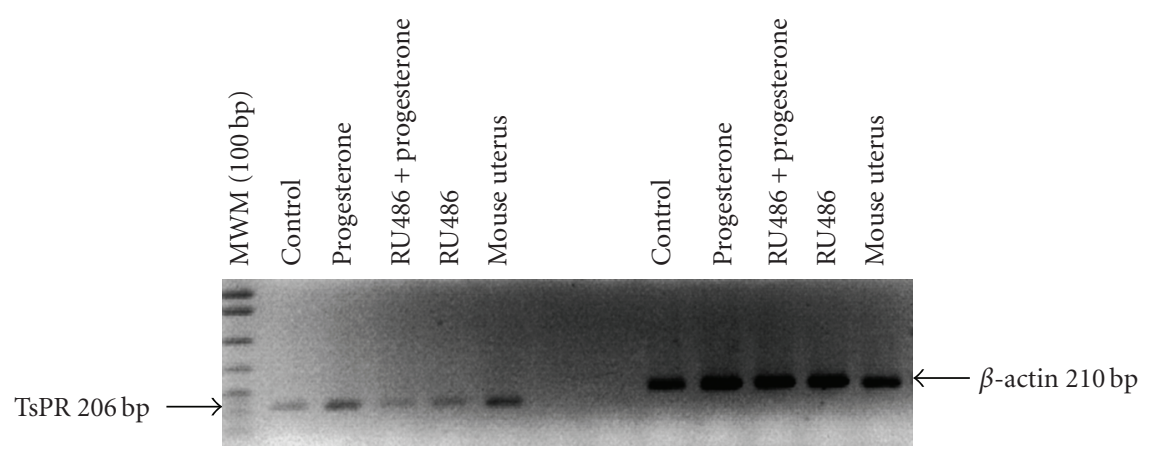

(a)

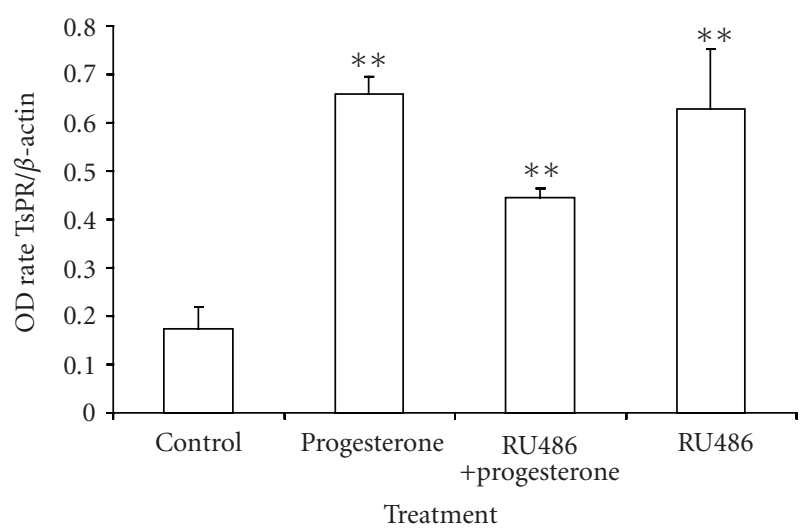

(b)

FIgURE 3: TsPR gene expression in T. solium. A single band of $206 \mathrm{bp}$, corresponding to the Taenia solium Progesterone Receptor (TsPR) was detected in T. solium and mouse uterus (a). Progesterone as well as RU486 increased TsPR expression in T. solium cysticerci. Densitometric analysis is shown in (b). $\beta$-actin was used as constitutive expression gene. Data are represented as mean $+/-\mathrm{SD} ;{ }^{* *} P<.05$.

The opposed effect was observed with RU486 treatment: when T. solium cysticerci were exposed to $2.5 \mu \mathrm{M}$ and higher concentrations of RU486, a total inhibition of worm development was seen, even in the presence of the highest concentration of progesterone (Figure 2(a)). Dissimilar to the results of evagination percentage, the effect of RU486 on worm length was independent of the tested concentration. In addition, the T. solium worm gradually grew up in response to $0.25 \mu \mathrm{M}$ of progesterone (Figure 2(b)). Differentiated worms in absence of hormones or anti-hormonal stimulus had a spontaneous development, reaching their maximum length $(3.5 \mathrm{~mm})$ at 12 day in culture (Figure $2(\mathrm{~b})$ ). Once again, in the presence or absence of progesterone, no worm differentiation was observed with $2.5 \mu \mathrm{M}$ of RU486 along all the time of in vitro culture (Figure 2(b)).

A single band corresponding to the expected molecular weight of the amplified fragment of PR (approximately $206 \mathrm{bp}$ ) was detected from TsPR and mouse uterus (Figure 3(a)). Moreover, progesterone and RU486, separately and/or combined, increased TsPR mRNA content related to the control group (Figure 3(b)).

TsPR protein was detected by western blot as a main band of approximately $116 \mathrm{KDa}$ (Figure $4(\mathrm{a})$ ). This TsPR matches to PR-B isoform that has been previously reported for rodents and human cell lines [43-46]. Nevertheless, no bands corresponding to PR-A isoform were identified in any of the tested treatments in the parasite (Figure 4(a)). On the contrary, by using the same antibody, a couple of bands of 87 and $116 \mathrm{KDa}$ were well recognized in the control tissue used (rat uterus), corresponding to PR-A and PRB, respectively (Figure 4(a)). TsPR content was increased in response to RU486 but not when parasites were exposed to both progesterone and RU486 (Figure 4(b)).

A preliminary sequence of TsPR was obtained by PCR product sequencing and then translated to protein sequence (ExPASy Proteomics Server). A posterior analysis of this TsPR showed homology of around $60 \%$ to the protein sequences previously reported for mouse, rat, rabbit, and human PR in the GenDataBank. It is important to mention that the analyzed conserved motif was situated in a region of approximately 50 aa, located in the DNA-binding domain of the C-terminal motif (from aa position number 110 to 160 of the mammal sequences described earlier) (data not shown). A more precise analysis of the TsPR sequence involved a Neighbor Joint Tree (NJT) for studying phylogenetic relationships (Figure 5). This NJT was inferred from the PR dataset, producing a single tree composed by 5 groups. The first contained sequences of 6 species of mammals (including pig and human, both natural hosts of the parasite), the second group consisted of one reptilian and one bird. The third group included only one amphibian. The fourth group was composed of 3 fish species, and the fifth group was 

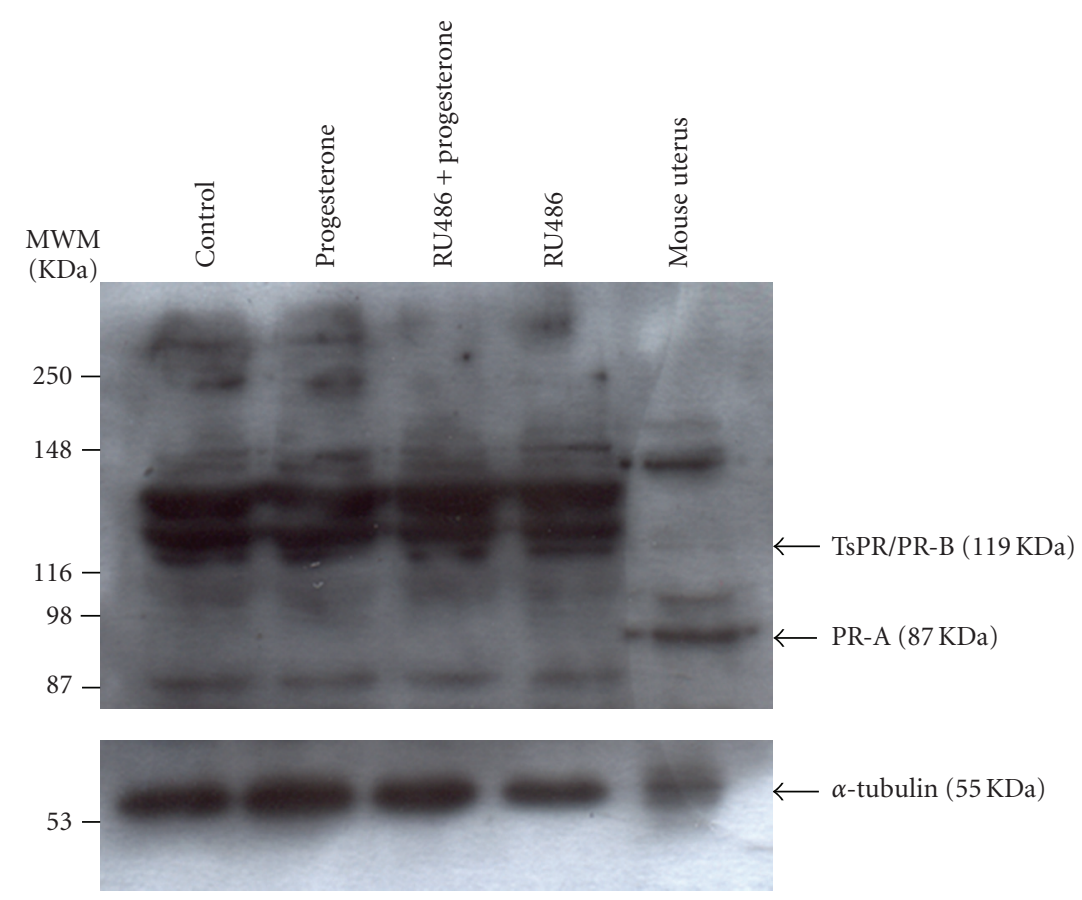

(a)

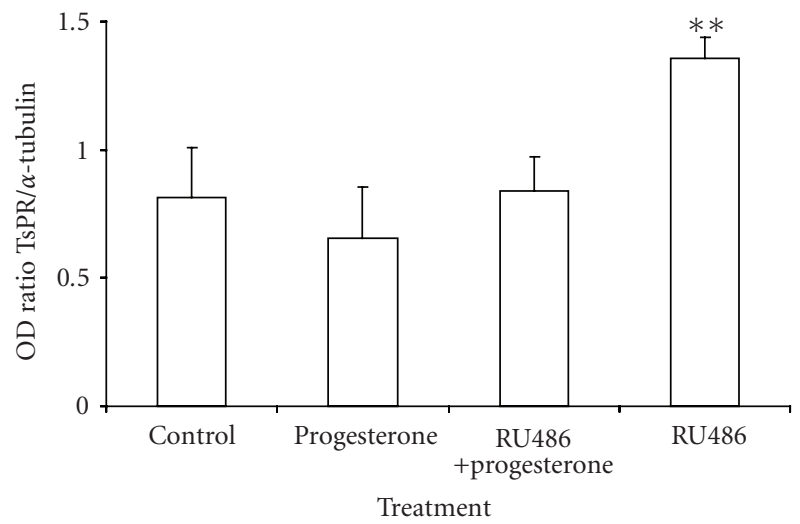

(b)

FIGURe 4: TsPR protein detection in T. solium. A main band of approximately $116 \mathrm{KDa}$, equivalent to TsPR was detected in T. solium (a). This TsPR matches to the molecular weight of PR-B isoform from rat uterus, but not to PR-A ( $\sim 87 \mathrm{KDa})$, where no protein bands in the parasite were observed (a). TsPR protein was significantly up-regulated by RU486 alone, but not by progesterone and the combination between progesterone and RU486 (b). $\alpha$-tubulin was used as control constitutive protein. Data are represented as mean $+/-\mathrm{SD}$; ${ }^{* *} P<.05$.

only composed by T. solium. The phylogenetic relationships among the 5 groups received good bootstrap support ranging from 66 to $100 \%$ (see Figure 5). Additionally, TsPR is related to the PR family of vertebrates, more closely associated to reptilian and amphibian (Figure 5). This finding suggests that TsPR is definitively not a product of host cell contamination, specifically not of pig nor human cells, because of the big distance between T. solium and mammals in the NJT.

\section{Discussion}

Here we describe the effects of progesterone and its antagonist RU486 upon scolex evagination and adult worm growth. First of all, it was clear that progesterone has a direct stimulatory effect on T. solium in vitro. In fact, progesterone exerts a marked evagination-promoting effect in a concentration-independent pattern, maintained entire time in culture, making that all parasites differentiate at 14 days of in vitro culture.

T. solium cysticerci not only showed evagination but they also presented a constant motility in the culture plate, which suggests that progesterone did not affect parasite viability and, therefore, they were alive during the culture process. Moreover, progesterone also induced the growth of the worm in the evaginated parasites by 2 folds, with respect to untreated cysticerci. 


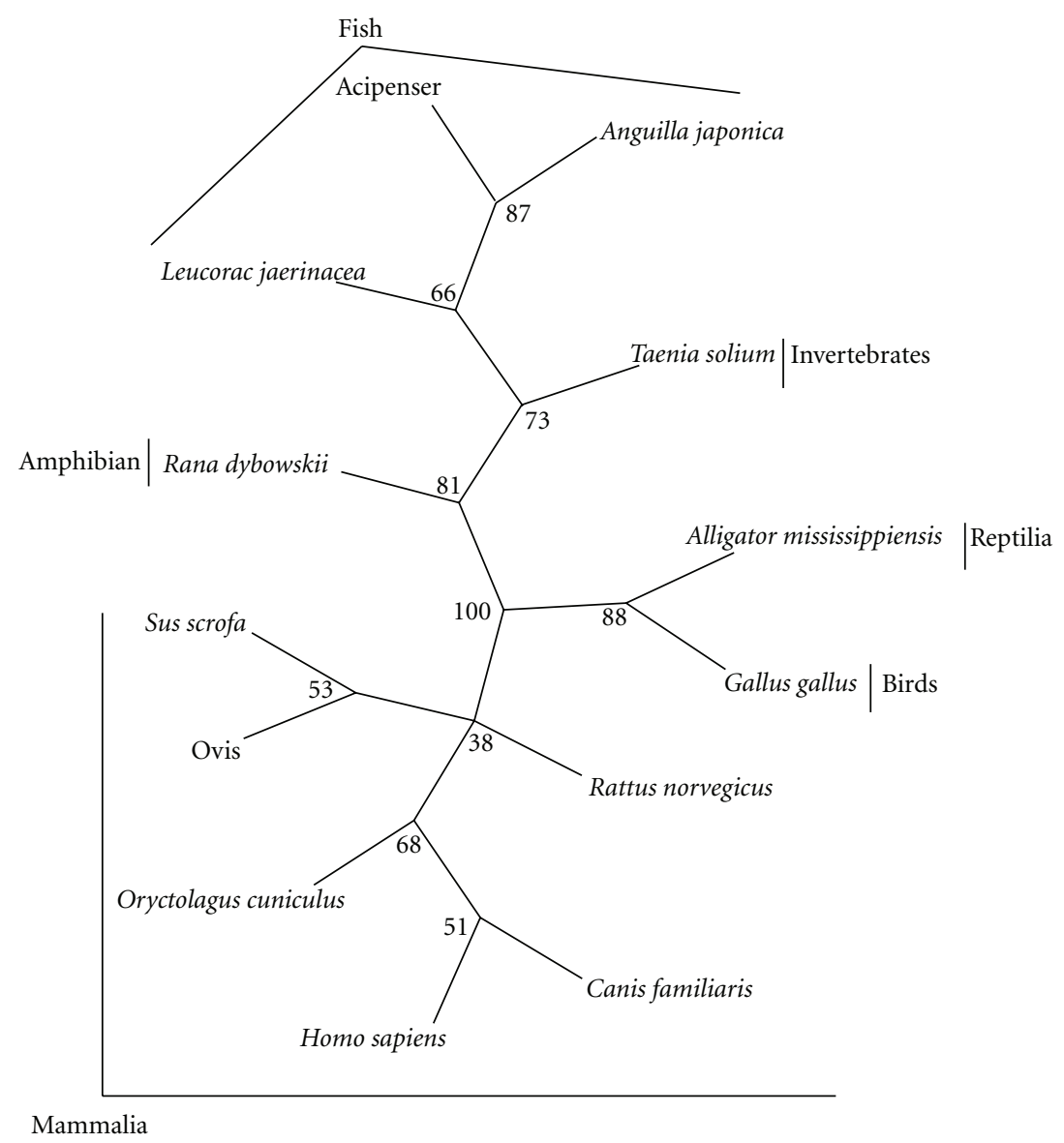

FIgURE 5: Neighbor Join Tree (NJT) for phylogenetic relation analysis. PRs from several species of fish, amphibian, reptilian, bird, and mammals were analyzed through a NJT for searching probable relationship to the T. solium PR identified and sequenced. TsPR showed close relation to PRs from fish and amphibian, but distant to their counterparts in mammals. Numbers on the NJT means bootstrap support ranging among analyzed species.

Interestingly, neither evagination nor worm size depend on progesterone concentrations, which are different from those tipically found in mammals [47-50]. This finding highlights that sex steroids could conserve several effects on invertebrate organisms (such as helminth parasites), but their action mechanisms may differ from those reported in rodent models and human cells.

We also provide elements for a possible action mechanism through which progesterone exerts its effects upon T. solium differentiation. In fact, this helminth seems to have developed a molecule able to recognize progesterone and mediate its effects, a protein we named TsPR (Taenia solium Progesterone Receptor). This putative steroid receptor is expressed in T. solium and was upregulated by progesterone and RU486.

It is important to highlight that TsPR has a counterpart sequence, recently identified in the sequencing of the $T$. solium genome, performed by The Consortium of the Taenia solium Genome Project of the Universidad Nacional Autónoma de México (data not shown) [51]. These additional data confirm the existence of a PR in T. solium.
Besides, TsPR was detected both at mRNA and protein levels. Interestingly, this TsPR matches only to PR-B isoform, suggesting that $T$. solium presents only one form of PR-like, as it has been also described in other organisms such as rabbits $[52,53]$. This result indicates that a single form of TsPR is actively expressed and translated to protein, which probably has repercussions on T. solium physiology and its relationship to the host.

Furthermore, this TsPR showed high degree of relation to their PR counterparts in fish and amphibian, but it is distant to mammalian sequences. This finding has two important connotations: firstly, it suggests that TsPR is a close relative of the steroid nuclear receptors that bind to progesterone. Secondly, this PR in T. solium definitively is not a contamination product from pig or human cells because it has a far relation to PRs sequenced in these organisms.

RU486 $(2.5 \mu \mathrm{M})$ totally inhibited scolex evagination and consequently worm development. This remarkable antiparasite effect was maintained even in the presence of the highest concentration of progesterone $(63.5 \mu \mathrm{M})$. Moreover, RU486-treated cysticerci showed internal disorganization; 
development of opaque areas in the tegument, loss of translucence in the vesicule, and no differentiation along all the in vitro culture time. This result suggests a possible toxic effect of RU486 upon T. solium cysticerci, independent of the TsPR that has not yet been reported for this progesteroneantagonist.

RU486 induced TsPR expression as well as the concomitant protein production, clearly suggesting that this antihormone regulates PR expression in T. solium, as it has been reported in rodent models and human cell lines [54, 55]. Then, although RU486 had upregulatory effects on TsPR, the same toxic response in T. solium was observed, indicating that RU486's antiparasite mechanism does not involve TsPR nor progesterone-dependent pathways inhibition, but a potent cysticide effect on the parasite, worthy of further investigation.

\section{Conclusions}

Here, we describe two different effects of progesterone and RU486 that probably have different action mechanisms directly upon T. solium development. Progesterone effects could explain, at least partially, the higher prevalence of cysticercosis in pregnant than in nonpregnant swine [19]. Then, T. solium could respond to progesterone through a TsPR with capacity to bind the hormone, and in this way regulate parasite viability and survival inside an immunocompetent host. To the opposite, the RU486 antiparasite effect was lethal for T. solium. In fact, RU486 permanently blocked the evagination of $T$. solium cysticerci, which has negative consequences to the adult tapeworm development and production of infective eggs. Thus, RU486 could be considered as a potential new agent in the interruption of the T. solium's life cycle, once we already know optimal doses of this antihormone as well as adverse and secondary effects in humans and other animal models.

To our knowledge, this is the first report where anticysticercus effects are described for RU486 and open a promissory field in the design of new strategies that include the antihormone therapy in the control of taeniasis/cysticercosis caused by Taenia solium.

\section{Acknowledgments}

Financial support was provided by Grants no. 58283 from CONACyT, Grant no. IN-213108 from the Programa de Apoyo a Proyectos de Innovación Tecnológica, Dirección General de Asuntos del Personal Académico, UNAM, and a special Grant from the Fundación Miguel Alemán-SSA all of them to J. Morales-Montor. Also, G. Escobedo has a posdoctoral fellowship from the Direccion General de Asuntos del Personal Académico, UNAM. Isabel Pérez Montfort corrected the English version of this manuscript.

\section{References}

[1] T. E. Nash, G. Singh, A. C. White, et al., "Treatment of neurocysticercosis: current status and future research needs," Neurology, vol. 67, no. 7, pp. 1120-1127, 2006.
[2] H. H. Garcia and O. H. Del Brutto, "Neurocysticercosis: updated concepts about an old disease," The Lancet Neurology, vol. 4, no. 10, pp. 653-661, 2005.

[3] A. Flisser, E. Sarti, M. Lightowlers, and P. Schantz, "Neurocysticercosis: regional status, epidemiology, impact and control measures in the Americas," Acta Tropica, vol. 87, no. 1, pp. 4351, 2003.

[4] A. C. White Jr., "Neurocysticercosis: a major cause of neurological disease worldwide," Clinical Infectious Diseases, vol. 24, no. 2, pp. 101-113, 1997.

[5] P. C. Fan and W. C. Chung, "Sociocultural factors and local customs related to taeniasis in east Asia," The Kaohsiung Journal of Medical Sciences, vol. 13, no. 11, pp. 647-652, 1997.

[6] A. E. Gonzalez, C. Gavidia, N. Falcon, et al., "Protection of pigs with cysticercosis from further infections after treatment with oxfendazole," The American Journal of Tropical Medicine and Hygiene, vol. 65, no. 1, pp. 15-18, 2001.

[7] Z. Pawlowski, J. Allan, and E. Sarti, "Control of Taenia solium taeniasis/cysticercosis: from research towards implementation," International Journal for Parasitology, vol. 35, no. 11-12, pp. 1221-1232, 2005.

[8] C. Barton Behravesh, L. F. Mayberry, J. R. Bristol, et al., "Population-based survey of taeniasis along the United StatesMexico border," Annals of Tropical Medicine and Parasitology, vol. 102, no. 4, pp. 325-333, 2008.

[9] A. C. Moore, L. I. Lutwick, P. M. Schantz, et al., "Seroprevalence of cysticercosis in an Orthodox Jewish community," The American Journal of Tropical Medicine and Hygiene, vol. 53, no. 5, pp. 439-442, 1995.

[10] P. M. Schantz, A. C. Moore, J. L. Munoz, et al., "Neurocysticercosis in an Orthodox Jewish community in New York City," The New England Journal of Medicine, vol. 327, no. 10, pp. 692695, 1992.

[11] M. T. Rabiela, Y. Hornelas, C. Garcia-Allan, E. Rodriguez-delRosal, and A. Flisser, "Evagination of Taenia solium cysticerci: a histologic and electron microscopy study," Archives of Medical Research, vol. 31, no. 6, pp. 605-607, 2000.

[12] G. Escobedo, L. Lopez-Griego, and J. Morales-Montor, "Neuroimmunoendocrine modulation in the host by helminth parasites: a novel form of host-parasite coevolution?" NeuroImmunomodulation, vol. 16, no. 2, pp. 78-87, 2009.

[13] O. Bottasso and J. Morales-Montor, "Neuroimmunomodulation during infectious diseases: mechanisms, causes and consequences for the host," NeuroImmunomodulation, vol. 16, no. 2, pp. 65-67, 2009.

[14] H. S. Thabet, S. S. Thabet, N. M. Ali, and N. S. Ahmed, "Effects of ovariectomy and thyroidectomy on course and outcome of Trichinella spiralis infection in rat," Journal of the Egyptian Society of Parasitology, vol. 38, no. 1, pp. 29-46, 2008.

[15] Y. Osorio, D. L. Bonilla, A. G. Peniche, P. C. Melby, and B. L. Travi, "Pregnancy enhances the innate immune response in experimental cutaneous leishmaniasis through hormonemodulated nitric oxide production," Journal of Leukocyte Biology, vol. 83, no. 6, pp. 1413-1422, 2008.

[16] C. D. Santos, M. P. Toldo, F. H. Santello, V. Filipin Mdel, V. Brazao, and J. C. do Prado Junior, "Dehydroepiandrosterone increases resistance to experimental infection by Trypanosoma cruzi," Veterinary Parasitology, vol. 153, no. 3-4, pp. 238-243, 2008.

[17] J. Morales-Montor, G. Escobedo, J. A. Vargas-Villavicencio, and C. Larralde, "The neuroimmunoendocrine network in the complex host-parasite relationship during murine cysticercosis," Current Topics in Medicinal Chemistry, vol. 8, no. 5, pp. 400-407, 2008. 
[18] N. Pena, J. Morales, J. Morales-Montor, et al., "Impact of naturally acquired Taenia solium cysticercosis on the hormonal levels of free ranging boars," Veterinary Parasitology, vol. 149, no. 1-2, pp. 134-137, 2007.

[19] J. Morales, T. Velasco, V. Tovar, et al., "Castration and pregnancy of rural pigs significantly increase the prevalence of naturally acquired Taenia solium cysticercosis," Veterinary Parasitology, vol. 108, no. 1, pp. 41-48, 2002.

[20] F. W. Bazer, R. C. Burghardt, G. A. Johnson, T. E. Spencer, and $\mathrm{G}$. Wu, "Interferons and progesterone for establishment and maintenance of pregnancy: interactions among novel cell signaling pathways," Reproductive Biology, vol. 8, no. 3, pp. 179-211, 2008.

[21] G. Escobedo, C. Larralde, A. Chavarria, M. A. Cerbon, and J. Morales-Montor, "Molecular mechanisms involved in the differential effects of sex steroids on the reproduction and infectivity of Taenia crassiceps," Journal of Parasitology, vol. 90, no. 6, pp. 1235-1244, 2004.

[22] L. I. Terrazas, R. Bojalil, T. Govezensky, and C. Larraide, "A role for 17- $\beta$-estradiol in immunoendocrine regulation of murine cysticercosis (Taenia crassiceps)," Journal of Parasitology, vol. 80, no. 4, pp. 563-568, 1994.

[23] J. Morales-Montor, S. Baig, C. Hallal-Calleros, and R. T. Damian, "Taenia crassiceps: androgen reconstitution of the host leads to protection during cysticercosis," Experimental Parasitology, vol. 100, no. 4, pp. 209-216, 2002.

[24] J. A. Vargas-Villavicencio, C. Larralde, and J. Morales-Montor, "Gonadectomy and progesterone treatment induce protection in murine cysticercosis," Parasite Immunology, vol. 28, no. 12, pp. 667-674, 2006.

[25] P. Ascenzi, A. Bocedi, and M. Marino, "Structure-function relationship of estrogen receptor $\alpha$ and $\beta$ : impact on human health," Molecular Aspects of Medicine, vol. 27, no. 4, pp. 299 402, 2006.

[26] R. A. Hiipakka and S. Liao, "Molecular mechanism of androgen action," Trends in Endocrinology and Metabolism, vol. 9, no. 8, pp. 317-324, 1998.

[27] S. Gadkar-Sable, C. Shah, G. Rosario, G. Sachdeva, and C. Puri, "Progesterone receptors: various forms and functions in reproductive tissues," Frontiers in Bioscience, vol. 10, pp. 2118 2130, 2005.

[28] C. Guerra-Araiza, A. Coyoy-Salgado, and I. Camacho-Arroyo, "Sex differences in the regulation of progesterone receptor isoforms expression in the rat brain," Brain Research Bulletin, vol. 59, no. 2, pp. 105-109, 2002.

[29] F. Remoue, J. C. Mani, M. Pugniere, A. M. Schacht, A. Capron, and G. Riveau, "Functional specific binding of testosterone to Schistosoma haematobium 28-kilodalton glutathione Stransferase," Infection and Immunity, vol. 70, no. 2, pp. 601605, 2002.

[30] C. Konrad, A. Kroner, M. Spiliotis, R. Zavala-Gongora, and $\mathrm{K}$. Brehm, "Identification and molecular characterisation of a gene encoding a member of the insulin receptor family in Echinococcus multilocularis," International Journal for Parasitology, vol. 33, no. 3, pp. 301-312, 2003.

[31] I. Bhai and A. K. Pandey, "Gonadal hormones in experimental Ancylostoma caninum infections in male Swiss albino mice," International Journal for Parasitology, vol. 12, no. 6, pp. 589591, 1982.

[32] J. Morales-Montor, F. Mohamed, A. M. Ghaleb, S. Baig, C. Hallal-Callerost, and R. T. Damian, "In vitro effects of hypothalamic-pituitary-adrenal axis (HPA) hormones on
Schistosoma mansoni," Journal of Parasitology, vol. 87, no. 5, pp. 1132-1139, 2001.

[33] G. Escobedo, C. W. Roberts, J. C. Carrero, and J. MoralesMontor, "Parasite regulation by host hormones: an old mechanism of host exploitation?" Trends in Parasitology, vol. 21, no. 12, pp. 588-593, 2005.

[34] R. T. Damian, "Parasite immune evasion and exploitation: reflections and projections," Parasitology, vol. 115, supplement, pp. S169-S175, 1997.

[35] M. Shibayama, J. Serrano-Luna Jde, S. Rojas-Hernandez, R. Campos-Rodriguez, and V. Tsutsumi, "Interaction of secretory immunoglobulin A antibodies with Naegleria fowleri trophozoites and collagen type I," Canadian Journal of Microbiology, vol. 49, no. 3, pp. 164-170, 2003.

[36] M. Spiliotis, C. Konrad, V. Gelmedin, et al., "Characterisation of EmMPK1, an ERK-like MAP kinase from Echinococcus multilocularis which is activated in response to human epidermal growth factor," International Journal for Parasitology, vol. 36, no. 10-11, pp. 1097-1112, 2006.

[37] K. Brehm and M. Spiliotis, "The influence of host hormones and cytokines on Echinococcus multilocuiaris signalling and development," Parasite, vol. 15, no. 3, pp. 286-290, 2008.

[38] G. W. Esch and J. D. Smyth, "Studies on the in vitro culture of Taenia crassiceps," International Journal for Parasitology, vol. 6, no. 2, pp. 143-149, 1976.

[39] J. Morales-Montor, G. Escobedo, M. Rodriguez-Dorantes, N. Tellez-Ascencio, M. A. Cerbon, and C. Larralde, "Differential expression of AP-1 transcription factor genes c-fos and c-jun in the helminth parasites Taenia crassiceps and Taenia solium," Parasitology, vol. 129, no. 2, pp. 233-243, 2004.

[40] J. D. Thompson, D. G. Higgins, and T. J. Gibson, "CLUSTAL $\mathrm{W}$ : improving the sensitivity of progressive multiple sequence alignment through sequence weighting, position-specific gap penalties and weight matrix choice," Nucleic Acids Research, vol. 22, no. 22, pp. 4673-4680, 1994.

[41] J. Felsenstein, PHYLIP (Phylogeny Inference Package), Version 3.572, University of Washington, Seattle, Wash, USA, 1999.

[42] D. L. Swofford, PAUP 4.0610. Phylogenetic Analysis Using Parsimony (and Other Methods), Sinauer, Sunderland, Mass, USA, 2002.

[43] E. Cabrera-Munoz, A. Gonzalez-Arenas, M. Saqui-Salces, et al., "Regulation of progesterone receptor isoforms content in human astrocytoma cell lines," The Journal of Steroid Biochemistry and Molecular Biology, vol. 113, no. 1-2, pp. 8084, 2009.

[44] A. Gonzalez-Arenas, T. Neri-Gomez, C. Guerra-Araiza, and I. Camacho-Arroyo, "Sexual dimorphism in the content of progesterone and estrogen receptors, and their cofactors in the lung of adult rats," Steroids, vol. 69, no. 5, pp. 351-356, 2004.

[45] I. Camacho-Arroyo, G. Gonzalez-Aguero, A. GamboaDominguez, M. A. Cerbon, and R. Ondarza, "Progesterone receptor isoforms expression pattern in human chordomas," Journal of Neurooncology, vol. 49, no. 1, pp. 1-7, 2000.

[46] I. Camacho-Arroyo, C. Guerra-Araiza, and M. A. Cerbon, "Progesterone receptor isoforms are differentially regulated by sex steroids in the rat forebrain," Neuroreport, vol. 9, no. 18, pp. 3993-3996, 1998.

[47] O. A. Sukocheva, Y. Yang, and J. F. Gierthy, "Estrogen and progesterone interactive effects in postconfluent MCF-7 cell culture," Steroids, vol. 74, no. 4-5, pp. 410-418, 2009.

[48] C. L. Gibson, L. J. Gray, P. M. Bath, and S. P. Murphy, "Progesterone for the treatment of experimental brain injury; a systematic review," Brain, vol. 131, no. 2, pp. 318-328, 2008. 
[49] E. R. Norwitz, V. Snegovskikh, F. Schatz, et al., "Progestin inhibits and thrombin stimulates the plasminogen activator/inhibitor system in term decidual stromal cells: implications for parturition," American Journal of Obstetrics and Gynecology, vol. 196, no. 4, pp. e381-e388, 2007.

[50] C. L. Butts, S. A. Shukair, K. M. Duncan, et al., "Progesterone inhibits mature rat dendritic cells in a receptor-mediated fashion," International Immunology, vol. 19, no. 3, pp. 287296, 2007.

[51] H. Aguilar-Diaz, R. J. Bobes, J. C. Carrero, et al., "The genome project of Taenia solium," Parasitology International, vol. 55, supplement, pp. S127-S130, 2006.

[52] H. Loosfelt, F. Logeat, M. T. Vu Hai, and E. Milgrom, "The rabbit progesterone receptor. Evidence for a single steroidbinding subunit and characterization of receptor mRNA," The Journal of Biological Chemistry, vol. 259, no. 22, pp. 1419614202, 1984.

[53] I. Camacho-Arroyo, A. M. Pasapera, and M. A. Cerbon, "Regulation of progesterone receptor gene expression by sex steroid hormones in the hypothalamus and the cerebral cortex of the rabbit," Neuroscience Letters, vol. 214, no. 1, pp. 25-28, 1996.

[54] J. C. Leo and V. C. Lin, "The activities of progesterone receptor isoform $\mathrm{A}$ and $\mathrm{B}$ are differentially modulated by their ligands in a gene-selective manner," International Journal of Cancer, vol. 122, no. 1, pp. 230-243, 2008.

[55] A. Shatnawi, T. Tran, and M. Ratnam, "R5020 and RU486 act as progesterone receptor agonists to enhance Sp1/Sp4dependent gene transcription by an indirect mechanism," Molecular Endocrinology, vol. 21, no. 3, pp. 635-650, 2007. 

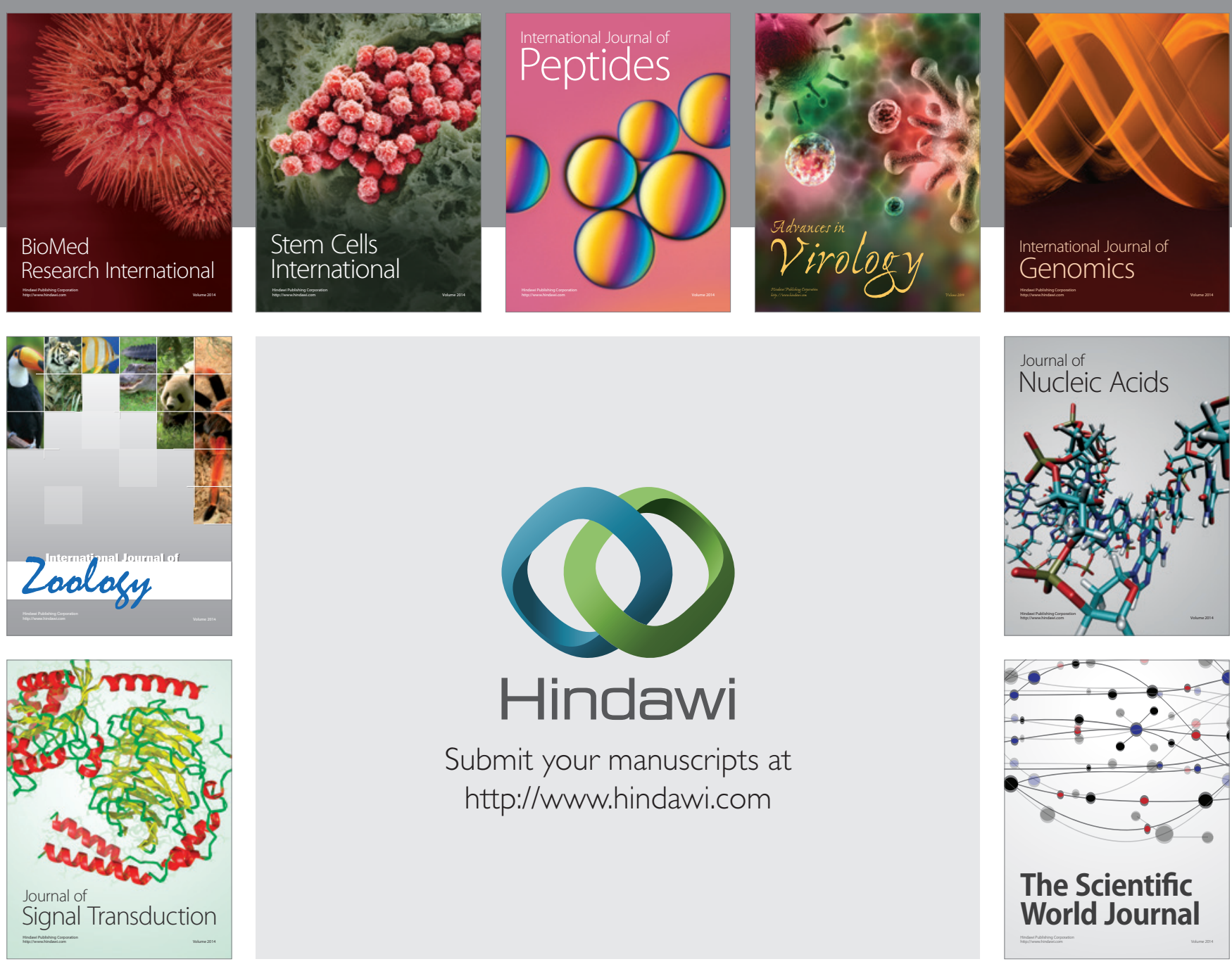

Submit your manuscripts at

http://www.hindawi.com
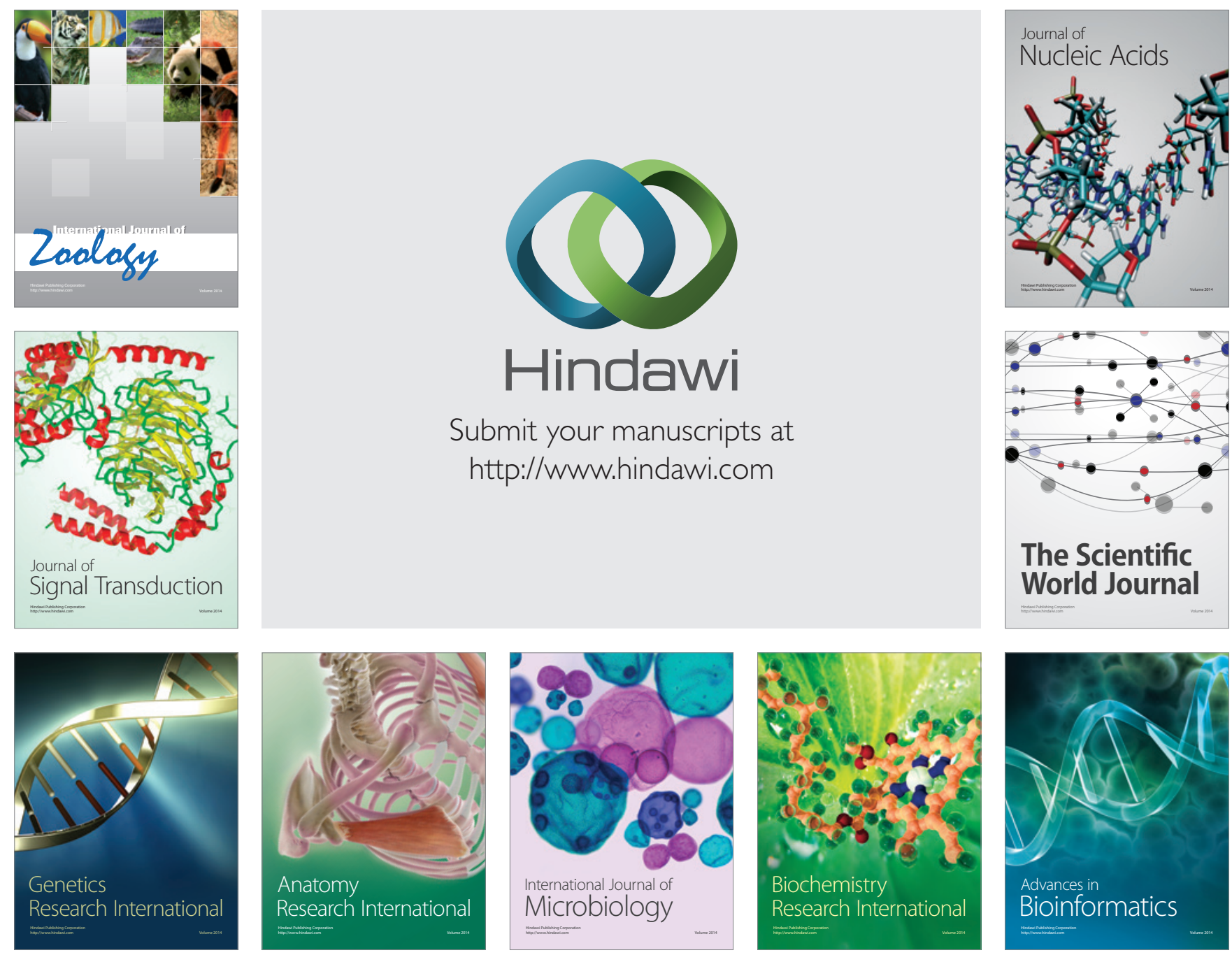

The Scientific World Journal
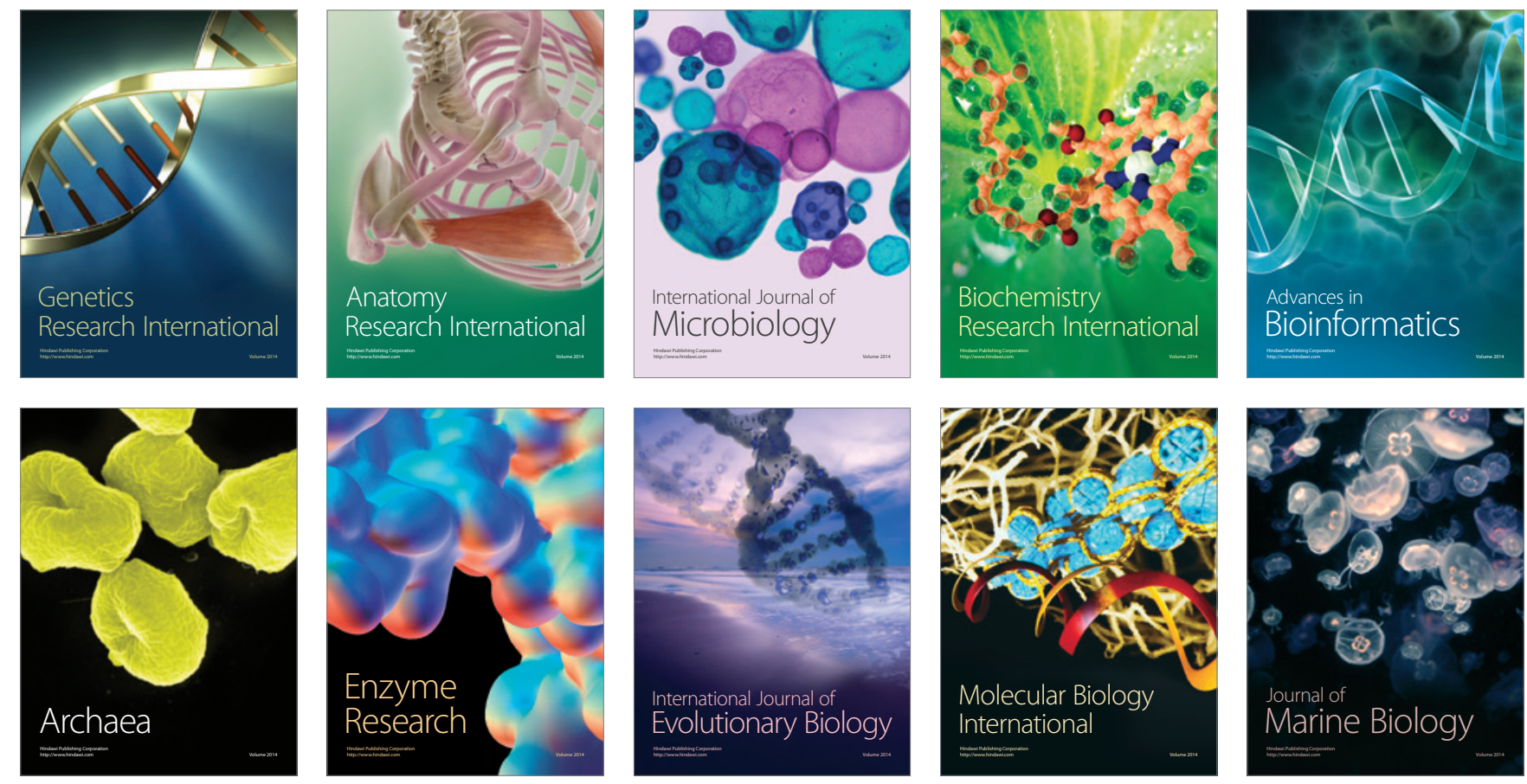